\title{
Glomerulopatía del trasplante. Resultados clínicos y seguimiento de casos
}

\author{
Citlalli Orizaga-de la Cruz, Renato Parra-Michel, Venice Chávez-Valencia, Francisco Fuentes-Ramírez, \\ Yuritomo Aragaki, Isela Márquez-Magaña y Martha Arisbeth Villanueva-Pérez \\ Servicio de Nefrología, Hospital General Regional No. 46, Instituto Mexicano del Seguro Social (IMSS), Guadalajara, Jal., México
}

\section{Resumen}

Objetivo: Describir la evolución de la función del injerto en pacientes con glomerulopatía del trasplante mediante los valores de creatinina, proteinuria y tasa de filtrado glomerular estimada. Método: Estudio descriptivo y transversal, realizado en el Hospital General Regional No. 46 del Instituto Mexicano del Seguro Social. Incluyó pacientes con trasplante renal y diagnóstico de glomerulopatía del trasplante por biopsia del injerto renal realizado entre el 1 de enero de 2006 y el 31 de abril de 2013. Se registraron la creatinina sérica, la proteinuria y la tasa de filtrado glomerular estimada al diagnóstico y a los 6 , 12 y 24 meses. Se muestran los resultados con números, porcentajes y desviaciones estándar. Resultados: De los 42 pacientes incluidos, a los 6 meses del diagnostico el 14\% con deterioro en la función del injerto y el $7.1 \%$ con pérdida de la función; a los 12 meses, el 17.9\% con pérdida de la función del injerto; a los 24 meses, el 36.3\% con pérdida en la función del injerto y vuelta a la diálisis. Conclusiones: La evolución de nuestros pacientes parece ser mejor que en otras series de casos descritas en la literatura.

PALABRAS CLAVE: Glomerulopatía del trasplante. Función del injerto renal. Trasplante renal.

\begin{abstract}
Objective: We describe the evolution of graft function in patients with transplant glomerulopathy measure by levels of serum creatinine, proteinuria and estimated glomerular filtration rate. Method: Cross-sectional study conducted in the Regional General Hospital No. 46 IMSS. Included patients with kidney allograft and diagnosis of renal biopsy of transplant glomerulopathy grafting between January 1, 2006 to April 31, 2013 serum creatinine, proteinuria and estimated glomerular filtration rate at diagnosis, 6, 12 and 24 was recorded months. The results are shown with numbers, percentages and standard deviations. Results: 42 patients were included. At 6 months of diagnosis, 14\% decline in graft function and $7.1 \%$ graft loss. At 12 months, $17.9 \%$ graft loss, and at 24 months $36.3 \%$ had chronic graft dysfunction and graft loss as return to dialysis. Conclusions: Evolution in our patients seems to be better to other series of cases reported in the literature.
\end{abstract}

KEY WORDS: Transplant glomerulopathy. Mexico. Kidney allograft function. Kidney transplant.

\author{
Correspondencia: \\ Citlalli Orizaga-de la Cruz \\ Avda. Río Nilo, 3975 \\ Col. Villas del Nilo \\ C.P. 44824, Guadalajara, Jal., México \\ E-mail: cicoriz@gmail.com
}

Fecha de recepción en versión modificada: 22-10-2016

Fecha de aceptación: 01-11-2016

DOI://dx.doi.org/10.24875/GMM.17002367
Gac Med Mex. 2017;153:747-751

Contents available at PubMed www.gacetamedicademexico.com 


\section{Introducción}

La enfermedad renal crónica es la resultante de diversas enfermedades cronicodegenerativas y está considerada una enfermedad catastrófica debido al número creciente de casos, por los altos costos de inversión, limitados recursos de infraestructura y humanos, detección tardía y altas tasas de morbilidad y mortalidad en los programas de sustitución ${ }^{1}$, siendo el trasplante renal el tratamiento de elección².

Sin embargo, dentro las principales causas de pérdida de los injertos renales están la disfunción crónica del injerto y la muerte del paciente con injerto funcionante por causas cardiovasculares ${ }^{3}$. Al realizar una biopsia con indicación clínica, la principal causa de falla del injerto es secundaria a rechazo mediado por anticuerpos (humoral), en pacientes con pobre adherencia al tratamiento inmunosupresort.

La glomerulopatía del trasplante (GT) ha recibido mucha atención en los últimos años como una manifestación de rechazo humoral crónic ${ }^{5,6}$. La GT es una condición patológica única limitada a injertos renales ${ }^{7}$, descrita por Porter, et al. en 1967 y caracterizada por un engrosamiento o una duplicación de la membrana basal glomerular (en ausencia de otras condiciones que puedan causar este patrón histológico $)^{5-7}$. Con frecuencia es diagnosticada en etapas tardías postrasplante y se asocia con una pobre sobrevida del injerto ${ }^{1,5,7-9}$.

Varios estudios han investigado el significado pronóstico de diferentes parámetros (tanto clínicos como histológicos) en relación con la GT, en un intento de identificar un subgrupo de pacientes con una progresión de la enfermedad más lenta y determinar el grupo de pacientes con necesidad de terapia inmunomoduladora $^{7}$. El promedio de sobrevida estimado a los 5 años posbiopsia con el diagnóstico de GT es de solo el $16.7 \%^{10}$.

Es importante destacar que la reducción en la tasa de filtrado glomerular (TFG), una vez realizada la biopsia renal, tiende a disminuir. Cosio, et al. ${ }^{8}$ encontraron que, tras un año de seguimiento, el $27 \%$ de los pacientes con diagnóstico de GT tenían ya una disminución de la TFG del $50 \%$.

Hasta la fecha no se cuenta con una terapia efectiva para la $\mathrm{GT}^{5,7,11}$. Existe fuerte evidencia de que el control de la presión arterial y la inhibición de la angiotensina II son medidas efectivas y disminuyen la progresión de la enfermedad ${ }^{5}$. Además, se ha sugerido que la inmunosupresión adicional podría ser útil, esto basado en la patogenia y en casos aislados de manejo, por lo que sigue considerándose evidencia preliminar $^{5}$. Se ha utilizado el cambio de esquema inmunosupresor (asociación de tacrolimus y micofenolato de mofetilo), con una disminución mantenida en los títulos de anticuerpos? ${ }^{7}$.

Recientes estrategias con esta misma finalidad se basan en el uso de recambios plasmáticos, inmunoglobulina intravenosa y agentes depletores de células $T$ y $B$, incluyendo globulina antilinfocitos o rituximab, pero los resultados no han sido tan buenos como se esperaba, sin lograr definir aún el protocolo óptimo de tratamiento ${ }^{11,12}$. El $50 \%$ de los pacientes tratados con rituximab presentan pérdida del injerto en un periodo de 26 meses, con estabilización de los valores de creatinina sérica $(\mathrm{CrS})$ y disminución importante en la proteinuria del $50 \%$ restante a 3 años de seguimiento ${ }^{13}$.

El objetivo del estudio fue conocer la evolución a 6 , 12 y 24 meses de la función del injerto en pacientes con diagnóstico de GT mediante los valores de CrS, proteinuria y TFG estimada (TFGe).

\section{Método}

Estudio descriptivo y transversal realizado en el Hospital General Regional No. 46 del Instituto Mexicano del Seguro Social en Guadalajara, Jalisco. De los pacientes con trasplante renal en seguimiento en la consulta externa, se incluyeron aquellos con GT cuyo diagnóstico se estableció entre el enero de 2006 y abril del 2013, que cumplieron los criterios de inclusión: edad entre 16 y 60 años, cualquier sexo, que contaran con reporte de biopsia de injerto renal con descripción de microscopía óptica, inmunofluorescencia y C4d por tinción inmunohistoquímica, en la que se estableció el diagnóstico de GT, así como determinación de proteinuria y CrS basal, a los 6, 12 y 24 meses, de acuerdo con el tiempo de seguimiento.

Se eliminaron pacientes con reporte de biopsia de injerto renal incompleta, sin especificar el tratamiento recibido en la nota médica, que perdieron seguimiento de la consulta externa de nefrología, y que no contaban con reporte de CrS y proteinuria en las notas médicas.

Para determinar la evolución se registraron la creatinina y la proteinuria en el momento basal y a los 6 , 12 y 24 meses después del diagnostico de GT, mismas que se realizaron de forma sistemática en el seguimiento de los pacientes con trasplante renal. Además, se estimó la TFG basal y a los 6, 12 y 24 
meses por la fórmula MDRD-4 (Modification of Diet in Renal Disease 4).

La evolución se concluyó como: a) sin deterioro en la función del injerto: incremento en la CrS menor del $50 \%$ del valor basal o disminución en la TFGe $<10 \mathrm{ml} / \mathrm{min}$; b) con deterioro en la función del injerto: incremento en la $\mathrm{CrS}$ mayor del $50 \%$ del valor basal o disminución en la TFGe $>10 \mathrm{ml} / \mathrm{min}$; o c) pérdida de la función del injerto: TFGe $<10 \mathrm{ml} / \mathrm{min}$, vuelta a la diálisis o retrasplante renal.

\section{Análisis estadístico}

Las variables numéricas se muestran como promedios con desviaciones estándar; las variables nominales se muestran como números y porcentajes. Se realizó estadística descriptiva con frecuencias absolutas. Se utilizó el programa SPSS versión 15 en español.

\section{Resultados}

De los 906 pacientes en seguimiento de trasplante renal, 42 con diagnóstico de GT cumplieron con los criterios de inclusión, por lo que se calculó una frecuencia de GT en esta población del $4.6 \%$. El tiempo promedio desde el trasplante renal hasta el diagnóstico fue de $90.8 \pm 41.7$ meses (Tabla 1). Los 42 pacientes cumplieron un mínimo de tiempo de 6 meses tras el diagnóstico de GT, 39 pacientes a los 12 meses y solo 33 a los 24 meses.

Se muestran los resultados de la evolución de la GT (Tabla 2), así como los valores de creatinina, proteinuria y TFGe por MDRD-4 a lo largo del tiempo de seguimiento (Tabla 3 ).

El promedio de tiempo hasta la pérdida del injerto tras el diagnóstico es de 16.35 meses (rango: 6-44 meses). La sobrevida del injerto fue del $92.8 \%$, el $82.05 \%$ y el $63.6 \%$ a los 6,12 y 24 meses, respectivamente.

Del total de los pacientes, 18 recibieron tratamiento; en el $66.6 \%$ se realizó cambio a tacrolimus, y en el resto se instauró terapia a base de agentes biológicos. Es importante mencionar que el $72.2 \%$ de los pacientes que recibieron algún tipo de tratamiento se mantuvo con el injerto funcionante hasta los 24 meses de seguimiento.

La principal indicación de realización de biopsia fue la elevación de la creatinina en 28 pacientes (66.7\%); sin embargo, el $57.14 \%$ presentaban también proteinuria en el momento del diagnóstico.
Tabla 1. Variables demográficas

\begin{tabular}{|c|c|c|c|}
\hline \multicolumn{3}{|l|}{ Edad, años $(\bar{\chi} \pm s)$} & $26.2 \pm 8.7$ \\
\hline \multicolumn{3}{|c|}{ Sexo masculino/femenino, n/n (\%) } & $29 / 13(31 / 69)$ \\
\hline \multicolumn{3}{|l|}{ Peso $(\mathrm{kg}) \bar{\chi} \pm \mathrm{s}$} & $61.9 \pm 14.50$ \\
\hline \multicolumn{3}{|l|}{ TRDVR, n (\%) } & $34(81)$ \\
\hline \multicolumn{3}{|l|}{ TRDMC, n (\%) } & $5(11.9)$ \\
\hline \multicolumn{3}{|l|}{ TRDVA, n (\%) } & $3(7.1)$ \\
\hline \multicolumn{3}{|l|}{ Antecedente rechazo, n (\%) } & $19(45)$ \\
\hline \multicolumn{3}{|l|}{ HAS, n (\%) } & $3(7.1)$ \\
\hline \multicolumn{3}{|l|}{$\mathrm{DM}, \mathrm{n}(\%)$} & $2(4.8)$ \\
\hline \multicolumn{3}{|l|}{ Cr habitual $(\mathrm{mg} / \mathrm{dl}) \bar{\chi} \pm s$} & $1.54 \pm 0.53$ \\
\hline \multicolumn{3}{|l|}{ Cr al diagnóstico $(\mathrm{mg} / \mathrm{dl}) \bar{\chi} \pm \mathrm{s}$} & $2.19 \pm 0.95$ \\
\hline \multicolumn{3}{|c|}{ Proteinuria al diagnóstico $(\mathrm{g} / 24 \mathrm{~h}) \bar{\chi} \pm \mathrm{s}$} & $3.35 \pm 2.6$ \\
\hline \multicolumn{3}{|c|}{ TFGe diagnóstico $(\mathrm{ml} / \mathrm{min}) \bar{\chi} \pm \mathrm{s}$} & $42.2 \pm 20.9$ \\
\hline \multicolumn{4}{|c|}{$\begin{array}{l}\text { Cr: creatinina; DM: diabetes mellitus; HAS: hipertensión arterial sistémica; } \\
\text { TFGe: tasa de filtrado glomerular estimada; TRDMC: trasplante renal de donador en } \\
\text { muerte cerebral; TRDVA: trasplante renal de donador vivo afectivo; TRDVR: trasplante } \\
\text { renal de donador vivo relacionado. }\end{array}$} \\
\hline Tiempo de aparición & 6 meses & 12 meses & 24 meses \\
\hline $\begin{array}{l}\text { Sin deterioro en la función, } \\
n(\%)\end{array}$ & $33(78.6)$ & $21(53.8)$ & $10(30.3)$ \\
\hline $\begin{array}{l}\text { Con deterioro en la función, } \\
n(\%)\end{array}$ & $6(14)$ & $11(28.2)$ & $11(33.3)$ \\
\hline Pérdida en la función, n (\%) & $3(7.1)$ & $7(17.9)$ & $12(36.3)$ \\
\hline
\end{tabular}

El hallazgo histopatológico más frecuente reportado es la fibrosis intersticial con atrofia tubular (IF/TA, interstitial fibrosis and tubular atrophy) en 28 pacientes, de los cuales en 14 era leve, en nueve era moderada y en cinco era grave; seguida de glomerulitis en el $54.8 \%$, C $4 d$ en el $40.5 \%$, tubulitis en el $28.6 \%$ y capilaritis en el $4.8 \%$.

Como análisis adicional se determinó la frecuencia de hallazgos histopatológicos y bioquímicos en el momento del diagnóstico que se relacionan con pobre sobrevida del injerto, tanto en pacientes con pérdida de la función del injerto como en los que continúan con injerto funcionante (Tabla 4).

\section{Discusión}

Se presenta el primer estudio realizado en el Hospital General Regional No. 46 con la finalidad de determinar la prevalencia y la evolución de los pacientes con GT, el cual cuenta con 906 pacientes en seguimiento de trasplante renal. 
Tabla 3. Valores de creatinina sérica, proteinuria de 24 horas y tasa de filtrado glomerular estimada en 42 casos

\begin{tabular}{|c|c|c|c|c|}
\hline Variable/tiempo de evolución & Al diagnóstico & 6 meses & 12 meses & 24 meses \\
\hline Creatinina sérica $(\mathrm{mg} / \mathrm{dl})^{*}$ & $2.1 \pm 0.94$ & $2.9 \pm 2.7$ & $2.5 \pm 1.55$ & $2.2 \pm 0.98$ \\
\hline Proteinuria $(\mathrm{g} / 24 \mathrm{~h})^{\star}$ & $3.3 \pm 2.60$ & $3.6 \pm 2.97$ & $2.6 \pm 1.8$ & $1.8 \pm 1.16$ \\
\hline Tasa de filtrado glomerular estimada (ml/min)* & $42.2 \pm 20.9$ & $37.4 \pm 19.8$ & $40.9 \pm 21.1$ & $43.7 \pm 25.4$ \\
\hline
\end{tabular}

*expresados como media \pm desviación estándar.

Tabla 4. Frecuencia de $\mathrm{C} 4 \mathrm{~d}$ positivo, IF/TA y proteinuria en rango nefrótico en pacientes con GT a los 24 meses de seguimiento

\begin{tabular}{lcc}
\hline & $\begin{array}{c}\text { Con pérdida } \\
\text { del injerto } \\
\mathbf{( n = 1 2 )}\end{array}$ & $\begin{array}{c}\text { Sin pérdida } \\
\text { del injerto } \\
(\mathbf{n}=\mathbf{3 0})\end{array}$ \\
\hline Proteinuria > 3.5 g/día, $\mathrm{n}(\%)$ & $6(50)$ & $5(16.6)$ \\
C4d positivo, $\mathrm{n}(\%)$ & $7(58.3)$ & $10(33.3)$ \\
IF/TA, $\mathrm{n}(\%)$ & $9(75)$ & $19(63.3)$ \\
\hline IF: fibrosis intersticial: TA: atrofia tubular & &
\end{tabular}

La literatura internacional reporta una prevalencia de GT del 1.6 al 20\%,10; esta variación tan amplia está en relación con el tipo de pacientes en que se realiza el diagnóstico. En nuestro estudio, la prevalencia de GT fue del $4.6 \%$, que si bien se encuentra dentro de lo reportado, tiende a ser baja, pero no podemos descartar un infradiagnóstico de la patología dado que solo incluimos pacientes con biopsias indicadas, y no por protocolo.

La GT se ha descrito con presentaciones tempranas como a solo dos meses del trasplante renal, aunque es más común de forma tardía. En este grupo de pacientes, el diagnóstico más temprano se realizó a los 21 meses del trasplante renal, con una media de 7.5 años; esto último está dentro de lo esperado, ya que si bien es mayor que lo reportado por autores como Shimizu, et al., ${ }^{14}$ cuyo diagnóstico es más temprano (4.4 años), estos incluyen en su estudio pacientes con GT a quienes se realizó biopsia tanto por deterioro en la función del injerto como por proteinuria o por protocolo, y no así en nuestro estudio, que incluyó solo pacientes con biopsias indicadas, lo cual influye en el tiempo hasta realizar el diagnóstico, pues se ha visto que hasta un $5 \%$ de los pacientes receptores de trasplante renal desarrollan esta lesión en el primer año postrasplante sin manifestaciones en la función del injerto renal que repercutan en el rango de sobrevida del injerto al momento del diagnóstico 8-10,13-15.

La forma de presentación más común en nuestros pacientes fue el deterioro en la función del injerto, con un incremento en la creatinina habitual del $40 \%$, siendo los valores séricos habituales de creatinina de $1.5 \pm 0.54 \mathrm{mg} / \mathrm{dl}$ y en el momento del diagnóstico de GT de $2.1 \pm 0.94 \mathrm{mg} / \mathrm{dl}$, lo que corresponde con lo reportado en otras series ${ }^{15,16}$.

La presencia de proteinuria en el $57.14 \%$ de los casos es también similar a lo descrito en literatura mundial, en la que oscila entre el 36 y el $93 \%^{14-16}$; sin embargo, se presentó proteinuria en rangos nefróticos en el $45.8 \%$ de los pacientes, lo cual es más del doble del valor reportado por Sun, et al. ${ }^{17}(18.6 \%)$, probablemente influenciado por el tipo de inmunosupresión utilizada en nuestros pacientes, ya que el $69 \%$ de ellos usan sirolimus, así como medidas antiproteinúricas que pudieran no haber sido usadas por los pacientes.

En nuestra serie, el hallazgo histopatológico más común es la IF/TA, aunque su frecuencia se encuentra por debajo de lo reportado, que va del 81 al $90 \%$ de los casos. La capilaritis y la glomerulitis que han descrito Shimizu, et al. ${ }^{14}$ dentro de los hallazgos más comunes asociados a la GT (84 y $81 \%$, respectivamente) no concuerdan con lo encontrado en el presente ni en otros estudios, en los que la frecuencia de ambas es menor, probablemente como resultado de la conocida variabilidad interobservador que existe en la interpretación de las biopsias ${ }^{14,17}$. Similar situación consideramos que ocurre con la tinción de $\mathrm{C} 4 \mathrm{~d}$, en la cual hay amplias variaciones de positividad, que van desde el 34 hasta el $69.7 \%$ en capilares peritubulares, dependiendo de la cohorte en cuestión; en nuestros casos, el C4d en capilares peritubulares se encuentra dentro de este rango ${ }^{14,16-18}$.

Existe fuerte evidencia respecto al mal pronóstico de la GT, pero los resultados en cuanto a la evolución son diversos, con reportes de pérdida del injerto desde un $60 \%$ a los 6 meses del diagnóstico hasta un $69.4 \%$ a los 26 meses, este último en un estudio de 4 años de seguimiento realizado por John, et al. ${ }^{10}$.

En el presente estudio, la pérdida del injerto es mucho menor que lo antes mencionado, pero mayor que la descrita por Cosio, et al., ${ }^{8}$ quienes tras 24 meses del diagnostico de GT reportan una pérdida del injerto menor del $20 \%$, pero con un aumento importante a los 
36 meses, cuando más del $50 \%$ de sus pacientes presentaron pérdida de injerto. Sin embargo, debemos tener en cuenta que en su estudio incluyeron pacientes con diagnóstico de GT realizado en biopsias protocolizadas, de tal forma que no existía deterioro inicial en la función del injerto, como en nuestros pacientes. En este mismo estudio encontraron que, tras un año de seguimiento, solo el 33\% mantenían una TFG estable, el $27 \%$ presentaron una disminución del $30 \%$, el $8 \%$ una disminución entre el 30 y el $50 \%$ y el $27 \%$ una disminución de la TFG del $50 \%$.

En nuestra serie de casos, al año de seguimiento el $28.2 \%$ de los pacientes presentaban deterioro en la función del injerto, el $53.8 \%$ se consideraron con función renal estable y el $17.9 \%$ tuvo pérdida del injerto, lo cual se incrementó al $36.3 \%$ a los 24 meses de seguimiento.

John, et al..$^{10}$ reportan una sobrevida del injerto a cinco años del 16.7\%; nosotros, a los seis meses, la encontramos del $92.8 \%$, a los 12 meses del $82.05 \%$ y a los 24 meses del $63.6 \%$, por lo que será importante continuar el seguimiento de estos pacientes, ya que la media de sobrevida se ha estimado en $43 \pm 7$ meses, por lo que podría esperarse una mayor pérdida de injertos en los siguientes dos años.

Diversos hallazgos, tanto histopatológicos como bioquímicos, se han relacionado con una peor sobrevida del injerto. Kieran, et al. ${ }^{19}$ concluyen en su estudio que el C4d por sí solo no predice la pérdida del injerto, pero sí cuando se encuentra junto con GT, siendo entonces un fuerte predictor de pérdida del injerto, con un riesgo relativo de 9.3. La combinación más poderosa para predecir la pérdida de injerto es GT, C4d positivo y $\mathrm{CrS}>2.3 \mathrm{mg} / \mathrm{dl}$.

Cosio, et al. ${ }^{5,8}$ han asociado la sobrevida y la función del injerto a la proteinuria en el momento del diagnóstico y la gravedad de la duplicación de membranas basales. Nosotros encontramos que los pacientes que presentaron durante el seguimiento pérdida del injerto tienen mayor frecuencia de proteinuria en rango nefrótico, IF/TA y C4d positivo en los capilares peritubulares.

Se muestra un incremento inicial importante en la proteinuria y la creatinina (y por ende se expresa en una menor TFG) en el momento del diagnóstico y hasta los seis meses de evolución, pero al año y a los 2 años de seguimiento la función renal parece estabilizarse e incluso mejorar. Esta mejoría en la caída de la TFG puede asociarse a una intensificación de la terapia inmunosupresora y la vigilancia del paciente, situación similar a la reportada por John, et al. ${ }^{10}$.
Es importante también tener en cuenta que el seguimiento en este estudio, como ya se mencionó, es menor que lo reportado como la media de sobrevida después del diagnóstico. Además, estamos conscientes de la pérdida a los 24 meses de seguimiento de un $21 \%$ de la muestra por no cumplir con el tiempo. Sin embargo, la evolución en nuestros pacientes parece ser mejor que la descrita en la literatura, con un importante número de injertos funcionantes a los 24 meses del diagnóstico de GT, por lo que será importante ampliar el tiempo de seguimiento.

\section{Financiamiento}

No hubo fuente de financiamiento externa.

\section{Bibliografía}

1. Méndez-Durán A, Méndez-Bueno JF, Tapia-Yáñez T, et al. Epidemiología de la insuficiencia renal crónica en México. Dial Traspl. 2010;31:7-11.

2. El Nahas M, Kawar BS, El Kossi M. Epidemiology, etiology, pathophysiology, and staging of chronic kidney disease. En: Lerma Edgar V, Nissenson Allen R, editors. Nephrology secrets. $3^{\text {rd }}$ ed. Philadelphia: Elsevier Mosby; 2012. p. 131-41.

3. Hernández Marrero D, González-Molina Alcaide M. Resultados globales del trasplante renal. En: Lorenzo SV, López GJM, editores. Nefrología al día. 2.a ed. Barcelona, España: Sociedad Española de Nefrología/ Plusmedical; 2012. (Consultado el 15 de agosto de 2013). Disponible en: http://www.revistanefrologia.com/es-publicacion-nefrologia-articulo-resultados-globales-del-trasplante-renal-XX342164212000058.

4. Sellarés J, de Freitas DG, Mengel M, et al. Understanding the causes of kidney transplant failure: the dominant role of antibody-mediated rejection and nonadherence. Am J Transplant. 2012;12:388-99.

5. Cosio FG, Gloor JM, Sethi S, et al. Transplant glomerulopathy. Am J Transplant. 2008:8:492-6.

6. Baid-Agrawal S, Farris $A B 3^{\text {rd }}$, Pascual M, et al. Overlapping pathways to transplant glomerulopathy: chronic humoral rejection, hepatitis $C$ infection, and thrombotic microangiopathy. Kidney Int. 2011;80:879-85.

7. Mohamed N, Avila-Casado C. Transplant glomerulopathy: pathogenesis, morphology, and prognosis. J Transplant Technol Res. 2011;S1:003.

8. Cosio FG, Grande JP, Wadei H, et al. Predicting subsequent decline in kidney allograft function from early surveillance biopsies. Am J Transplant. 2005;5:2464-72.

9. Gloor JM, Sethi S, Stegall MD, et al. Transplant glomerulopathy: subclinical incidence and association with alloantibody. Am J Transplant. 2007; 7:2124-32

10. John R, Konvalinka A, Tobar A, et al. Determinants of long-term graft outcome in transplant glomerulopathy. Transplantation. 2010;90:757-64.

11. Durrbach A, Francois $\mathrm{H}$, Beaudreuil $\mathrm{S}$, et al. Advances in immunosuppression for renal transplantation. Nat Rev Nephrol. 2010;6:160-7.

12. Crespo M, Oppenheimer F, Venetz JP, et al. Treatment of humoral rejection in kidney transplantation. Transplant Rev. 2006;20:95-103.

13. Rostaing L, Guilbeau-Frugier C, Fort M, et al. Treatment of symptomatic transplant glomerulopathy with rituximab. Transpl Int. 2009;22:906-13.

14. Shimizu T, Tanabe T, Shirakawa $H$, et al. Clinical and pathological analysis of transplant glomerulopathy cases. Clin Transplant. 2012;26(Suppl 24):37-42.

15. Sis B, Campbell PM, Mueller T, et al. Transplant glomerulopathy, late antibody-mediated rejection and the $A B C D$ tetrad in kidney allograft biopsies for cause. Am J Transplant. 2007;7:1743-52.

16. Perkowska-Ptasinska A, Ciszek M, Chmura A, et al. Transplant glomerulopathy: clinical and pathological correlations. Transplant Proc. 2009;41:141-9.

17. Sun $Q$, Huang $X$, Jiang $S$, et al. Picking transplant glomerulopathy out of the CAN: evidence from a clinico-pathological evaluation. BMC Nephrol. 2012;13:128.

18. Sijpkens YW, Joosten SA, Wong MC, et al. Immunologic risk factors and glomerular C4d deposits in chronic transplant glomerulopathy. Kidney Int. 2004;65:2409-18.

19. Kieran N, Wang X, Perkins J, et al. Combination of peritubular c4d and transplant glomerulopathy predicts late renal allograft failure. J Am Soc Nephrol. 2009;20:2260-8. 